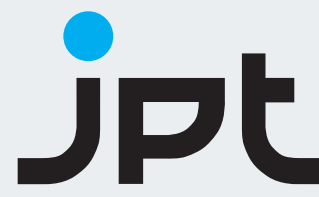

\title{
Antibody signatures defined by high-content peptide microarray analysis
}

\author{
Circulating antibodies are highly selective binding reagents directed to a vast repertoire of antigens. \\ Candidate antigens displayed as overlapping peptides on PepStar ${ }^{\mathrm{TM}}$ peptide microarrays can be \\ used to screen for recognition by serum antibodies from clinically well defined patient populations. \\ The methodology is robust and enables unbiased visualization of antigen-specific B-cell responses. \\ Additionally, autoantibody signatures of diagnostic value could be detected using microarrays \\ displaying thousands of human peptides.
}

\begin{abstract}
Antigen arrays have been used to gauge humoral immune responses. Using PepStar high-content peptide microarrays displaying overlapping peptides in the contexts of infectious and autoimmune diseases, allergy and cancer, we have improved the resolution of antigen arrays down to the epitope level.
\end{abstract}

A detailed knowledge of the target epitopes from pathogens recognized by $B$ cells from either vulnerable or protected individuals enables rational vaccine design and facilitates development of novel diagnostic tools. Currently, our knowledge of epitope-specific recognition patterns is limited to small regions of the proteomes of pathogens with large genomes. Recently, PepStar high-content peptide microarrays were used to profile antibody response to Mycobacterium tuberculosis epitopes $^{1,2}$ and allowed an unbiased and global view of the immune response, without the need to preselect target proteins. In that work, PepStar microarrays displaying 7,446 unique M. tuberculosis peptides together with 153 negative controls (printing buffer without peptides deposited), 24 positive controls (human immunoglobulins immobilized) and 96 fluorescent landmarks had been treated with sera from 34 individuals with active pulmonary tuberculosis and 35 healthy individuals to extract differences and antibody signatures predictive of tuberculosis. Linear 15-residue peptides derived from M. tuberculosis proteins and overlapping by 3 amino acids had been printed on functionalized glass slides, attached through a linker on the $\mathrm{N}$ terminus of the peptides. Resulting PepStar peptide microarrays can be stored at $4{ }^{\circ} \mathrm{C}$ for up to 18 months. Sera from subjects with active pulmonary tuberculosis were diluted 1:100 using a buffer consisting of PBS, 3\% FCS and 0.5\% Tween and placed onto the microarray surface $(300 \mu$ lotal volume, only $3 \mu \mathrm{l}$ of serum per slide), incubated at $4{ }^{\circ} \mathrm{C}$ for $16 \mathrm{~h}$, washed and dried, and

Mike Schutkowski, Johannes Zerweck, Antonia Masch \& Holger Wenschuh

JPT Peptide Technologies GmbH, Volmerstrasse 5, 12489 Berlin, Germany. Correspondence should be addressed to M.S. (schutkowski@jpt.com).
$\operatorname{IgA}$ and IgG peptide complexes were visualized using fluorescently labeled secondary anti-human $\lg \mathrm{A}$ or $\lg \mathrm{G}$ reagents.

In this analysis, three distinct patterns of antibody reactivity were identified: (i) peptides that were differentially recognized between tuberculosis-positive and healthy individuals, (ii) peptides that were exclusively recognized in all individuals with tuberculosis but not in any of the healthy ones, and (iii) peptides that were exclusively recognized in healthy but not tuberculosis-positive individuals. Antibody signatures in serum from tuberculosis-positive subjects from Armenia versus ones recruited in Sweden showed that lgG-defined tuberculosis epitopes are very similar in individuals with different genetic backgrounds. Notably, differentiation between tuberculosis-positive and healthy individuals does not cluster into specific recognition of distinct M. tuberculosis proteins, but into specific peptide epitope 'hotspots' at different locations within the same protein.

The use of PepStar peptide microarrays is not limited to the detection of antibody signatures directed against foreign antigens in infectious diseases ${ }^{1-3}$ and allergies ${ }^{4-6}$. Peptide microarrays displaying citrullinated peptide derivatives derived from human proteins enabled detection of induction of antigen-specific tolerance in multiple sclerosis after immunization with DNA encoding myelin basic protein in a randomized, placebo-controlled, phase $1 / 2$ a clinical trial ${ }^{7}$. The research used cerebrospinal fluid to probe autoantibody binding to PepStar microarray-bound peptides and demonstrated that the DNA vaccine produced cross-tolerance to other antigenic components, such as proteolipid protein. Moreover, PepStar microarrays displaying overlapping peptides derived from autoantigens are a useful tool to detect diagnostic autoantibody signatures in autoimmune diseases such as systemic lupus erythemotosus ${ }^{8}$.

Finally, peptide microarrays can be used for the reliable detection of autoantibodies with low serum titers, such as antibodies to tumorassociated antigens in individuals with cancer. We obtained a fluorescence image of a peptide microarray treated with serum from an 
APPLICATION NOTES
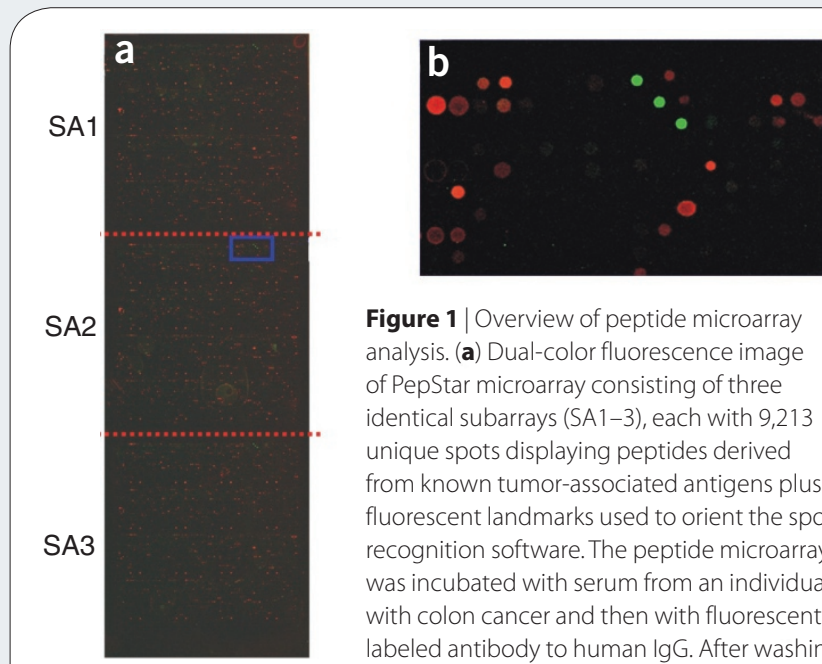

Figure 1 | Overview of peptide microarray analysis. (a) Dual-color fluorescence image of PepStar microarray consisting of three identical subarrays (SA1-3), each with 9,213 unique spots displaying peptides derived from known tumor-associated antigens plus 3 fluorescent landmarks used to orient the spotrecognition software. The peptide microarray was incubated with serum from an individual with colon cancer and then with fluorescently labeled antibody to human lgG. After washing and drying steps, the microarray was scanned

with standard microarray reader equipment (GenPix 4000B microarray scanner). (b) Close-up of the boxed region in $\mathbf{a}$, showing the three fluorescent landmarks (green spots) enabling correct orientation of the grid for spot recognition. Antibody-peptide antigen complexes were visualized using the appropriate red fluorescence-labeled secondary reagent. Red spots represent peptides with bound $\lg G$ antibody from the serum.

individual with colon cancer (Fig. 1). (Sera were obtained according to the rules and with the approval of the local ethics committees ("Gewebesammlung für molekularbiologische Untersuchungen bösartiger und gutartiger Erkrankungen des Gastrointestinaltraktes und endokriner Organe"; II HV 43/2004) and kindly supplied by M. Linnebacher, Section Molecular Oncology and Immune Therapy; University of Rostock, Germany.) The resulting antibody signatures from individuals with colon cancer together with the signatures from healthy individuals enable identification of unique peptide sequences for the development of new diagnostic or prognostic peptide chips.

\section{Conclusion}

PepStar high-content peptide microarrays are a robust platform for screening entire proteomes for antibody recognition. This has the potential to define biologically relevant targets in the context of rational vaccine design and development of novel diagnostics.

\section{ACKNOWLEDGMENTS}

This study was supported by Bundesministerium für Wirtschaft und Technology (InnoWatt grant IW051190).

1. Nahtman, T. et al. Validation of peptide epitope microarray experiments and extraction of quality data. J. Immunol. Methods 328, 1-13 (2007).

2. Gaseitsiwe, S. et al. Pattern recognition in pulmonary tuberculosis defined by high content peptide microarray chip analysis representing 61 proteins from M. tuberculosis. PLOS ONE 3, e3840 (2008).

3. Bongartz, J., Bruni, N. \& Or-Guil, M. (2009) Epitope mapping using randomly generated peptide libraries. Epitope Mapping Protocols 2nd ed., Methods in Molecular Biology 524 (eds. Reineke, U. \& Schutkowski, M.) ch. 17 (Humana Press; in the press).

4. Lin, J., Bardina, L. \& Shreffler, W.G. (2009) Microarrayed allergen molecules for diagnostics of allergy. Epitope mapping using randomly generated peptide libraries. Epitope Mapping Protocols 2nd ed., Methods in Molecular Biology 524 (eds. Reineke, U. \& Schutkowski, M.) ch. 19 (Humana Press; in the press).

5. Cerecedo, I. et al. Mapping of the lgE and lgG4 sequential epitopes of milk allergens with a peptide microarray-based immunoassay. J. Allergy Clin. Immunol. 122, 589-594 (2008)

6. Flinterman, A.E. et al. Peanut epitopes for lgE and IgG4 in peanut-sensitized children in relation to severity of peanut allergy. J. Allergy Clin. Immunol. 121, 737-743 (2008).

7. Bar-Or, A. et al. Induction of antigen-specific tolerance in multiple sclerosis after immunization with DNA encoding myelin basic protein in a randomized, placebo-controlled phase 1/2 trial. Arch. Neurol. 64, 1407-1415 (2007).

8. Lorenz, P., Kreutzer, M., Zerweck, J., Schutkowski, M. \& Thiesen, H.-J. (2009) Probing the epitope signatures of lgG antibodies in human serum from patients with autoimmune disease. Epitope mapping using randomly generated peptide libraries. Epitope Mapping Protocols 2nd ed., Methods in Molecular Biology 524 (eds. Reineke, U. \& Schutkowski, M.) ch. 18 (Humana Press; in the press).

This article was submitted to Nature Methods by a commercial organization and has not been peer reviewed. Nature Methods takes no responsibility for the accuracy or otherwise of the information provided. 